\title{
241: Malignant Catatonia Possibly Precipitated by SARS-CoV-2 Infection
}

\author{
Johnson, Sean ${ }^{1}$; Kapoor, Rajat ${ }^{2}$; Kim, Yo Sup ${ }^{3}$ \\ ${ }^{1}$ Indiana University School of Medicine, Indianapolis, IN \\ ${ }^{2}$ Indiana University Health Methodist Hospital, Indianapolis, IN \\ ${ }^{3}$ Indiana University SOM, Indianapolis, IN
}

Introduction: Malignant catatonia is a life-threatening condition that presents with fever, autonomic, delirium, and rigidity. We present a case of a 27 -year-old male with a past medical history of normal pressure hydrocephalous and polycythemia who was first admitted for depression and increasing catatonic symptoms.

Methods: The patient had no history of neuroleptic or serotonin agent usage prior to admission. During the outside hospital (OSH) admission, the patient became more catatonic and was subsequently transferred to a tertiary care center for electroconvulsive therapy (ECT) and further treatment. Upon admission, patient was found to be COVID negative. Brain magnetic resonance imaging, electroencephalography, lumbar puncture, serology testing for autoimmune encephalitis, Lyme, TSH, ESR, HIV, syphilis, Wilson's disease were all negative. Patient initially improved with scheduled Ativan and ECT with partial regain in motor function. However, overnight on hospital day 40, patient acutely spiked fevers to 40 degrees Celsius, had acute worsening in rigidity, and rapidly rising CPK level from 166 to 3200 in just over 48 hours. This patient was subsequently transferred to the Intensive Care Unit. Prior to progression to malignant catatonia patient had a COVID exposure from patient visitor. Repeat COVID testing was obtained the day patient was transferred and eventually resulted positive. In the following 8 days, the patient was intubated and placed on versed drip with Ativan IV with frequent ECT treatments without significant improvement.

Results: Malignant catatonia has been precipitated by a variety of viral, bacterial, and parasitic infections. However, malignant catatonia is primarily precipitated by CNS related infection. We report a case of a novel association of the SARS-CoV-2 viral infection with precipitation of malignant catatonia. This abstract was written prior to the discharge of patient, with treatment ongoing and outcome pending.

This is the author's manuscript of the work published in final form as:

Johnson, S., Kapoor, R., \& Kim, Y. S. (2021). 241: Malignant Catatonia Possibly Precipitated by SARS-CoV2 Infection. Critical Care Medicine, 49(1), 107. https://doi.org/10.1097/01.ccm.0000726852.54146.71 